\title{
Robotic Parathyroid Surgery: Current Perspectives and Future Considerations
}

\author{
Asit Arora ${ }^{a}$ George Garas b, c Neil Tolley ${ }^{b}$ \\ aDepartment of Otorhinolaryngology and Head and Neck Surgery, Guy's and St. Thomas' \\ NHS Foundation Trust, London, UK; ${ }^{b}$ Department of Otorhinolaryngology and Head and \\ Neck Surgery, St. Mary's Hospital, Imperial College Healthcare NHS Trust, London, UK; \\ 'Department of Surgical Research and Innovation, Royal College of Surgeons of England, \\ London, UK
}

\section{Keywords}

Robotic surgery · Parathyroid · Axillary approach · Evidence · Safety · Cosmesis · Policy

\begin{abstract}
Robotic parathyroidectomy represents a novel surgical approach in the treatment of primary hyperparathyroidism when the parathyroid adenoma has been pre-operatively localised. It represents the "fourth generation" in the evolution of parathyroid surgery following a process of surgical evolution from cervicotomy and 4-gland exploration to a variety of minimally invasive, open and endoscopic, targeted approaches. The existing evidence (levels 2-3) supports it as a feasible and safe technique with equivalent results to targeted open parathyroidectomy for primary hyperparathyroidism in carefully selected patients. However, it takes longer to perform and is more costly than conventional parathyroidectomy. It offers superior cosmesis by completely avoiding a neck scar making it a valid option for those patients who for biological and/or cultural reasons may wish to avoid a neck scar. Robotic parathyroidectomy is not for every patient, surgeon, or hospital. Its application should be confined to highvolume centres and experienced surgeons. Intensive training and proctorship are required for its safe implementation combined with careful patient selection. This particularly relates to the patient's body habitus (BMI $<30 \mathrm{~kg} / \mathrm{m}^{2}$ ) and concordance among the different imaging modalities used pre-operatively. With robotic market competition driving down costs, its role may change. For now, robotic parathyroidectomy occupies a niche role and can only be justified in a select subset of patients.

(c) 2018 S. Karger AG, Basel
\end{abstract}


Arora et al.: Robotic Parathyroid Surgery

\section{Introduction}

The incidence of primary hyperparathyroidism (pHPT) is estimated to be between 1 and 4 per 1,000 of the population making it the third commonest endocrine disorder after diabetes mellitus and osteoporosis [1]. pHPT has a preponderance for females (3:1 female-to-male ratio) with the majority ( $80 \%$ ) of cases being the result of a single functioning parathyroid adenoma. The remainder are attributed to parathyroid hyperplasia or, less commonly, multiple adenomas [2, 3].

Targeted parathyroidectomy is the indicated treatment for pHPT when the parathyroid adenoma has been localised pre-operatively. Various minimal and remote access techniques have been described, both open and endoscopic, though no approach has been shown to be overwhelmingly superior [4].

With the introduction of the da Vinci ${ }^{\circledR}$ surgical robot (Intuitive Surgical Inc., Sunnyvale, CA, USA), the latest addition to the armamentarium of parathyroid surgeons is robotic parathyroidectomy [5]. This approach overcomes the technical limitations associated with conventional endoscopic surgery whilst offering equivalent long-term results in terms of cure rates compared to the pre-existing techniques. Moreover, it achieves so without leaving the patient with any scar in the neck [5]. This review discusses the evolution of parathyroid surgery ultimately leading to the development of robotic parathyroidectomy, the evidence underlying this novel technique, its benefits and drawbacks, cost-effectiveness and value, current status, and possible future role.

\section{Evolution of Parathyroid Surgery: From "Exploratory" to "Targeted" Surgery}

The first successful parathyroidectomy was performed in 1925 in Vienna by Felix Mandel [6]. Over the subsequent decades, treatment involved 4-gland exploration, a procedure involving a cervicotomy, identification of all four parathyroid glands and resection of the macroscopically pathological one(s).

As with all surgical operations, with time, parathyroidectomy evolved. The decisive factor related to the introduction of sestamibi scintigraphy as a new localisation technique. Sestamibi scintigrams were shown to be significantly more accurate than pre-existing imaging modalities in localising the parathyroid adenoma [7].

This ability to accurately predict where the adenoma is located pre-operatively changed the ethos of parathyroidectomy from an "explorative" to a "targeted" approach, thus making unilateral surgery a valid option. Another important innovation related to the development of the quick intraoperative parathyroid hormone assay to assess surgical adequacy following resection of the abnormal gland(s) [8].

Numerous targeted parathyroidectomy techniques were subsequently described. Examples include endoscopic parathyroidectomy first described by Gagner in 1996 [9], minimally invasive parathyroidectomy via a lateral neck approach first described by Henry et al. in 1999 [10], and video-assisted parathyroidectomy first described by Miccoli et al. also in 1999 [11]. These targeted parathyroidectomy techniques were shown to produce excellent cure rates equivalent to 4-gland exploration and, as a result, were widely implemented [12]. However, they were all characterised by a common limitation, the presence of a neck scar (or more than one neck scars for certain endoscopic approaches).

Though a neck scar does not usually constitute a problem, there is a subset of patients that may have valid reasons for wanting to avoid this. This primarily relates to patients with a predisposition to hypertrophic scarring and keloid formation. Certain races, such as patients of Afro-Caribbean origin and those with dark skin pigmentation are especially prone to it. 
Arora et al.: Robotic Parathyroid Surgery

Moreover, in certain cultures, particularly in the Far East, the impact of a neck scar on body image and psyche can be detrimental $[13,14]$.

The above concerns relating to the presence of neck scar(s) led to the development of extracervical endoscopic parathyroidectomy. The first case was described by Ikeda et al. [15] in 2000 and involved an endoscopic transaxillary approach for pHPT and an anterior chest approach for secondary hyperparathyroidism. Though this novel technique completely avoided a neck scar through its translocation to a remote site (axilla or anterior chest), wider implementation was limited due to the constraints relating to endoscopic surgery in a remote and confined space, as is the neck. More specifically, limitations of endoscopic surgery involved the 2-dimensional visualisation, restriction in instrument movement, fulcrum effect, need for $\mathrm{CO}_{2}$ insufflation, and dependence on multiple assistants [16]. These limitations mandated the development of a new surgical platform that would enhance dexterity and improve visualisation whilst maintaining the key advantage of extracervical endoscopic surgery, the avoidance of a neck scar.

\section{Robotic Parathyroidectomy}

In an attempt to overcome the aforementioned limitations of endoscopic surgery, robotic technology was introduced in the early 2000s. This was achieved through the unique features of the da Vinci ${ }^{\circledR}$ surgical robot which includes a dual-channel endoscope offering simultaneous image magnification and depth perception through 3-dimensional visualisation combined with its wristed robotic instruments possessing 7 degrees of freedom, motion scaling, and tremor filtering, thus substantially enhancing surgical dexterity [17].

Following the wide uptake of robotic surgery among the various surgical specialties and the concurrently increasing experience with endoscopic parathyroidectomy and robotic thyroidectomy, robotic parathyroidectomy was the next advance $[18,19]$. This innovative technique representing the "fourth generation" in the evolution of parathyroid surgery was first described by Tolley et al. [5] in 2011 and involved a "scarless-in-the-neck" approach through an anterior chest (infraclavicular) incision and 3 trocars in the ipsilateral axillary line. In addition to the improved ergonomics compared to conventional endoscopic surgery, robotic parathyroidectomy does not require $\mathrm{CO}_{2}$ insufflation.

The preliminary results demonstrated that robotic parathyroidectomy was a safe and feasible alternative to the other targeted parathyroidectomy techniques [5]. With time, the technique evolved to require only one incision in the axilla. Subsequent studies followed to reproduce the results and compare robotic parathyroidectomy to its minimally invasive open counterpart.

\section{The Evidence Base}

In today's modern surgical practice, no discussion of a novel surgical technique would be complete without presenting the evidence [18]. Contrary to robotic thyroidectomy characterised by an extensive number of studies and reviews, the evidence for robotic parathyroidectomy is limited $[20,21]$.

Although robotic surgery has been performed successfully for the resection of both cervical and ectopic parathyroid adenomas, only the former are considered in this review. Ectopic parathyroid adenomas have been successfully resected with the da Vinci ${ }^{\circledR}$ surgical robot from a variety of anatomical locations including the mediastinum [22], pericardium [23], retropharyngeal space [24], and piriform sinus [25]. Thoracoscopic robotic approaches were used for the former two and transoral robotic surgery (TORS) for the latter two. 
Arora et al.: Robotic Parathyroid Surgery

Concerning the safety and feasibility of robotic parathyroidectomy there is level $2 \mathrm{~b}$ evidence to support its use as an alternative approach to targeted open parathyroidectomy in carefully selected patients. This includes both the anterior chest [5] and transaxillary approaches [26, 27].

In terms of robotic parathyroidectomy for the treatment of secondary and tertiary hyperparathyroidism (and thus non-localised disease), there is a single case series reporting successful total parathyroidectomy in six end-stage renal failure patients. It is important to state that in this study access was through the axillary-bilateral-breast approach [28]. Other approaches have also been described (for pHPT) including the retro-auricular approach although evidence for this approach is level 5 as the only existing studies are two case reports $[29,30]$.

There are two comparative studies in the literature evaluating robotic parathyroidectomy against its targeted open counterpart $[19,31]$. Both are non-randomised prospective studies with matched controls (evidence level 2). These two studies offer the only direct comparisons for operative outcomes and patient-reported outcome measures between robotic and open targeted parathyroidectomy with no indirect comparisons performed to date [32].

Operative time for robotic parathyroidectomy has been consistently shown to be significantly longer than that for its open counterpart (level 2). This is the case even after the learning curve has been surpassed. Despite this, no difference in mean blood loss or hospital stay has been demonstrated with all patients being discharged within $24 \mathrm{~h}$. Minor complications were observed in both series with one patient developing a superficial wound infection [19] and another a seroma [31]. Both were managed conservatively (the former with oral antibiotics), and no adverse clinical outcome occurred in either case.

The recurrent laryngeal nerve was identified and preserved in all cases with all patients having normal pre- and postoperative fibre-optic laryngoscopy [19, 31]. In terms of cure rates, robotic parathyroidectomy was shown to be as effective as targeted open parathyroidectomy (level 2). All adenomas were identified and removed successfully with histopathological evaluation confirming hypercellular parathyroid tissue in all excised specimens [19, 31 . With the exception of one patient [19], they all became normocalcaemic postoperatively and remained so during the follow-up period which exceeded 6 months.

With regard to flap-related complications - in view of the significantly larger area of subcutaneous dissection required for the robotic approach - the evidence is contradictory. Of the two existing comparative studies, one reported less pain for the robotic patients [19] whilst the other reported that three patients experienced "mild postoperative symptoms" relating to the flap [31]. This is likely to represent paraesthesia also described in the transaxillary robotic thyroidectomy literature [20]. There is no conclusive evidence with regards to postoperative pain for robotic parathyroidectomy compared to open surgery.

For postoperative quality of life, there is level 2 evidence that this is the same whether parathyroidectomy is performed robotically or conventionally [19]. Moreover, despite the anaerobic nature of the axilla, there is no evidence to support an increased infection rate over open surgery $[19,31]$.

As with all operations, the importance of patient selection cannot be emphasised enough. Patients considered for robotic parathyroidectomy should have triple modality concordance with ultrasonography, sestamibi scintigraphy, and single-photon emission computed tomography to prevent failure and the subsequent need for 4-gland exploration via cervicotomy. Similarly, obese patients (BMI $>30 \mathrm{~kg} / \mathrm{m}^{2}$ ) are at higher risk of open conversion due to difficult access and thus should not be offered this approach (level 2). A full list of contraindications to robotic parathyroidectomy is presented in Figure 1. 
Fig. 1. Contraindications to transaxillary robotic parathyroid surgery. ASA, American Society of Anesthesiologists.

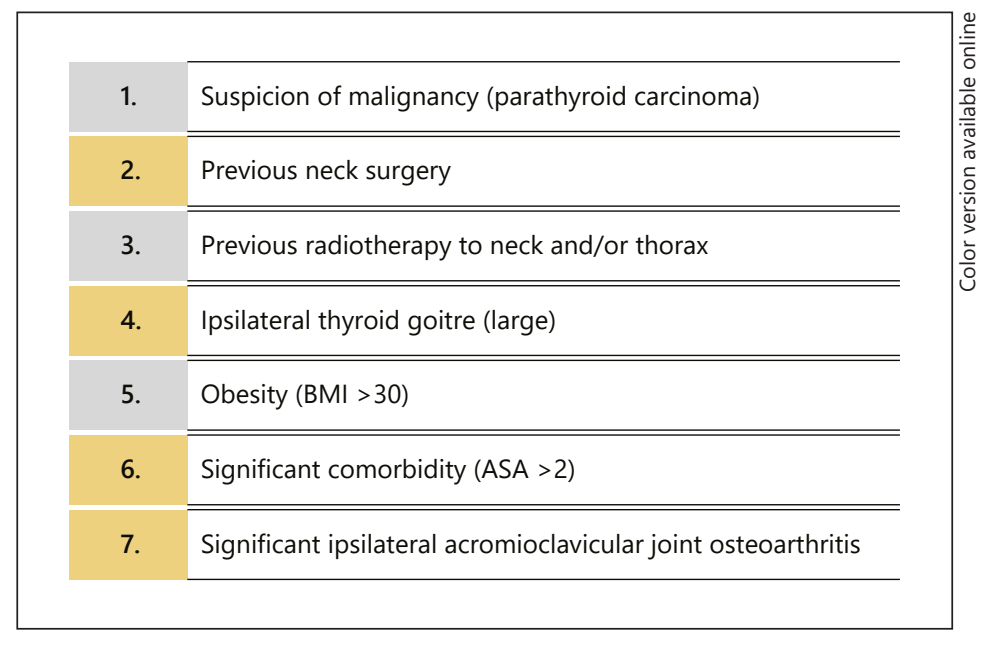

When looking at scar cosmesis, robotic parathyroidectomy appears to be superior to its open counterpart (level 2). However, this difference in patient satisfaction in favour of robotic parathyroidectomy is only present for up to 6 months. Beyond this time frame, no difference has been shown from studies with long-term prospective follow-up exceeding 2 years [19, 31].

\section{Potential Benefits}

Having established the safety and feasibility of robotic parathyroidectomy as well as its equivalence to targeted open surgery, the primary clinical advantage of the robotic approach appears to be the increased patient satisfaction achieved as a result of superior scar cosmesis. Robotic parathyroidectomy may therefore be justified in selected patients with a predisposition to hypertrophic scarring and/or keloid formation or those where the impact of a neck scar on body image could have detrimental effects on the individual's psyche often associated with social stigma $[4,13,19]$. As with all surgical operations, patient selection is paramount [33].

An additional benefit of robotic parathyroidectomy involves the combination of supreme visualisation and precise tissue manipulation (Fig. 2, 3) [34]. A 3-dimensional, magnified view, wristed robotic arms with 7 degrees of freedom, tremor filtering, and motion scaling are collectively exclusive to the da Vinci ${ }^{\circledR}$ surgical robot [5]. These features facilitate the precise identification and handling of vital structures in the confined space of the neck [19].

\section{Drawbacks}

Currently, robotic parathyroidectomy is associated with a number of drawbacks that limit its role. The longer operative time of robotic parathyroidectomy constitutes a major drawback compared to targeted open surgery [19]. Nonetheless, this does not appear to lead to any adverse patient outcomes. The same applies to the increased area of the subcutaneous flap raised, which in itself is the prime reason for the prolonged operative time - even after the surgeon has surpassed their learning curve - along with the time needed for docking the surgical robot [19]. 
Fig. 2. Intraoperative robotic console image illustrating blunt robotic dissection of the parathyroid adenoma.
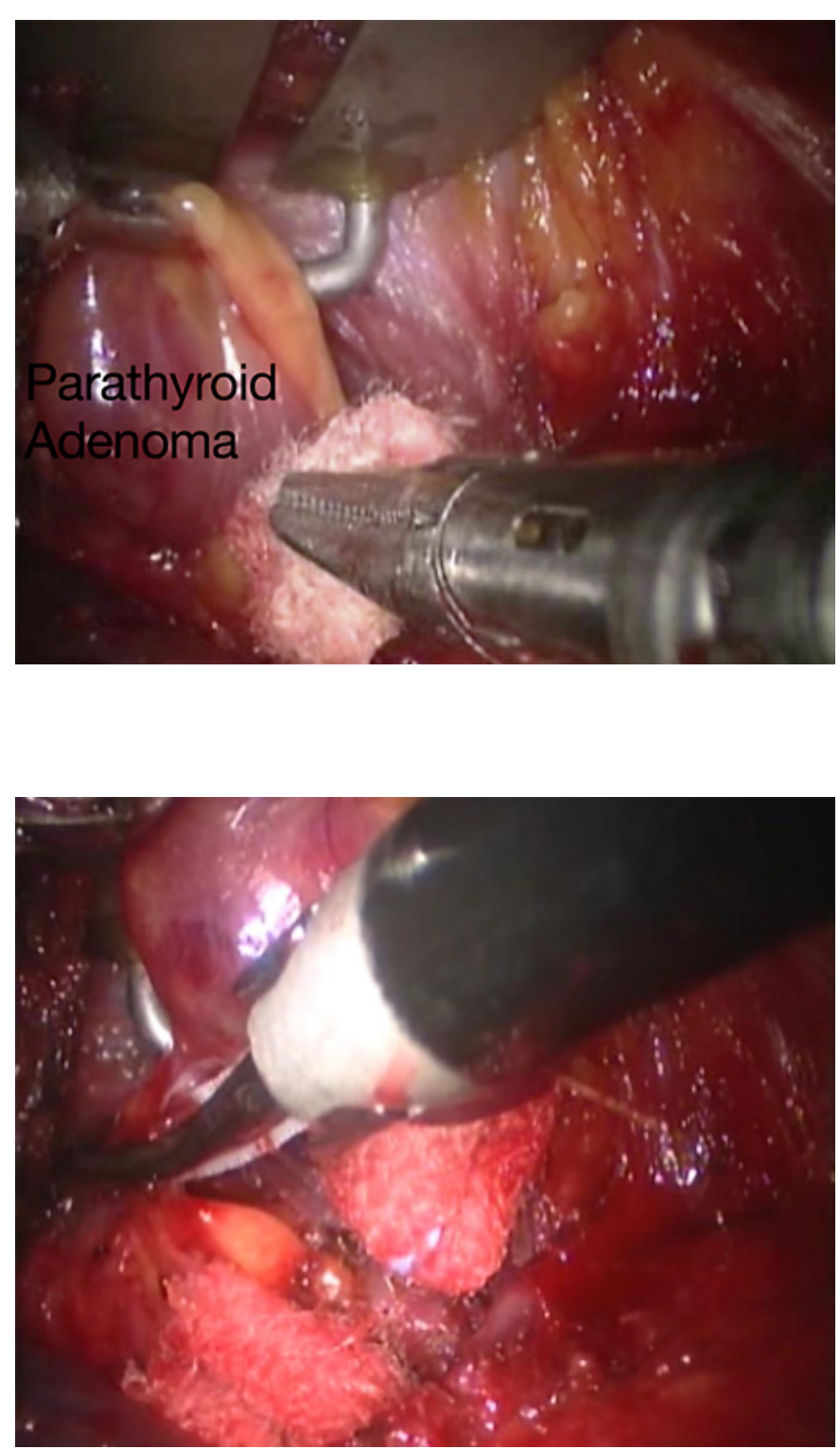

Fig. 3. Intraoperative robotic console image illustrating ligation of the vascular pedicle of the parathyroid adenoma with the harmonic scalpel ${ }^{\circledR}$ (Ethicon EndoSurgery Inc., Johnson \& Johnson, New Brunswick, NJ, USA).
An important drawback of transaxillary robotic surgery relates to the potential of brachial plexus injury $[35,36]$. Nevertheless, this complication has never been reported following transaxillary robotic parathyroidectomy - though such cases have been described (rarely) in the transaxillary robotic thyroidectomy literature [21,34]. Similarly, injury to the internal jugular vein, trachea, oesophagus, and thoracic duct has not been reported with transaxillary robotic parathyroidectomy but the possibility is there in view of the surgical anatomy and these complications having been encountered (rarely) with transaxillary robotic thyroidectomy. Thus, these constitute theoretical risks of transaxillary robotic parathyroidectomy, and patients should be counselled appropriately during the informed consent process. Furthermore, surgeons must be prepared for their occurrence and be competent in managing these complications despite their rarity [37]. Appropriate training and proctorship play an important role in achieving the safe implementation and development of skills. 


\section{Cost-Effectiveness and Value}

The National Institute for Health and Care Excellence (NICE) in the United Kingdom loosely applies a cost-effectiveness threshold of GBP 30,000 per quality-adjusted life year (QALY) in cases where efficacy is proven and GBP 20,000 per QALY in cases where clinical effectiveness is more controversial [38]. Based on this and the current high cost of robotic parathyroidectomy combined with the low level of evidence to support its use, robotic parathyroidectomy cannot be considered a cost-effective intervention at present [39]. However, the increasing competition in the robotic surgery market combined with the upcoming expiry of patents currently held by Intuitive Surgical Inc. are likely to reduce the costs associated with robotic surgery in the near future [40].

In the meantime, the largest barrier to adoption of robotic parathyroidectomy remains its prohibitive cost [5]. The cost of purchasing the da Vinci ${ }^{\circledR}$ surgical robot is GBP 1.5 million. Added to this are maintenance costs averaging GBP 120,000 per annum and surgical consumables at approximately GBP 1,300 per case [18]. Intuitive Surgical Inc. have successfully monopolised the surgical robotics market to date. Their business strategy has involved the acquisition of key competitors (e.g. Computer Motion Inc., Santa Barbara, CA, USA) and patenting of several robotic components [41].

A key driver for potentially reducing cost lies in developing robust market competition. The entry of multinational medical device companies such as Medtronic Inc. (Minneapolis, MN, USA), Medrobotics Inc. (Raynham, MA, USA), and the Johnson \& Johnson Inc. (New Brunswick, NJ, USA) - Google Inc. (Mountain View, CA, USA) partnership in the surgical robotics arena, as well as new "players" such as Cambridge Medical Robotics (Cambridge, UK) with the Versius ${ }^{\circledR}$ surgical robotic system and Titan Medical (Toronto, ON, Canada) with the SPORT ${ }^{\circledR}$ robot, is expected to make robotic surgical technology more widely affordable in the near future $[21,42]$.

Similar to robotic thyroid surgery, centralisation of robotic parathyroidectomy services to national high-volume centres and experienced surgeons is key. This will not only enhance patient outcomes, but also keep costs to a minimum through improved surgical efficiency and utilisation $[32,43,44]$.

\section{Current Status and Future Role}

At present, the experience with robotic parathyroidectomy is limited and confined to a handful of high-volume centres in the United Kingdom and United States. The existing evidence is not particularly high level (levels 2-3) but confirms that robotic parathyroidectomy is a feasible and safe technique with equivalent results to targeted open parathyroidectomy for pHPT in carefully selected patients [19, 21,33].

The unique advantage of completely avoiding a neck scar whilst overcoming the technical limitations of conventional endoscopic surgery, gives robotic parathyroidectomy a promising - though niche - role for those patients who for biological and/or cultural reasons may wish to avoid a neck scar.

\section{Conclusion}

Robotic parathyroidectomy represents the "fourth generation" in the evolution of parathyroid surgery. However, its uptake remains limited as it occupies a niche role in current surgical practice. The technique is only indicated in a small subset of patients with pHPT who 
Arora et al.: Robotic Parathyroid Surgery

for biological and/or cultural reasons may wish to avoid a neck scar. Its limited indications combined with the prohibitive cost, technical complexity, and limited evidence beyond safety and feasibility account for its minimal uptake. Upcoming advances in robotic surgical technology and a reduction in costs as a result of market competition may change this, making the technique more widely available. For now, the practice of robotic parathyroidectomy should be confined to high-volume centres and experienced surgeons ensuring careful patient selection and publishing their outcomes.

\section{Disclosure Statement}

Dr. George Garas, MD, FRCS holds a Royal College of Surgeons of England Doctoral Research Fellowship (Grant No. GG 1037600/2017-2018) and is also supported by Imperial College London (Grant No. CID 337755/2015-2018) and the Alexander S. Onassis Public Benefit Foundation (Grant No. F ZM 014-1/20162017). The funders had no role in the study design, data collection and analysis, decision to publish, or preparation of the manuscript. All authors declare no conflicts of any commercial interest.

\section{References}

1 Melton LJ 3rd: The epidemiology of primary hyperparathyroidism in North America. J Bone Miner Res 2002; 17:N12-N17.

2 Pallan S, Rahman MO, Khan AA: Diagnosis and management of primary hyperparathyroidism. BMJ 2012; 344:e1013.

-3 Venkat R, et al: Long-term outcome in patients with primary hyperparathyroidism who underwent minimally invasive parathyroidectomy. World J Surg 2012;36:55-60.

4 Christakis I, et al: Minimally invasive endocrine (thyroid, parathyroid, adrenal) surgery: evolution of operative techniques, safety, effectiveness and outcomes; in Hawthorne FT (ed): Minimally Invasive Surgery: Evolution of Operative Techniques, Safety and Effectiveness and Long-Term Clinical Outcomes. New York, Nova Science Publishers, 2014, pp 1-66.

5 Tolley N, et al: Robotic-assisted parathyroidectomy: a feasibility study. Otolaryngol Head Neck Surg 2011;144: 859-866.

6 Delbridge LW, Palazzo FF: First parathyroid surgeon: Sir John Bland-Sutton and the parathyroids. Aust NZ J Surg 2007;77:1058-1061.

7 Takami H, Ikeda Y, Wada N: Surgical management of primary hyperparathyroidism. Biomed Pharmacother 2000;54:17s-20s.

8 Nussbaum SR, et al: Intraoperative measurement of parathyroid hormone in the surgical management of hyperparathyroidism. Surgery 1988;104:1121-1127.

-9 Gagner M: Endoscopic subtotal parathyroidectomy in patients with primary hyperparathyroidism. Br J Surg 1996;83:875.

10 Henry JF, et al: Endoscopic parathyroidectomy via a lateral neck incision. Ann Chir 1999;53:302-306.

11 Miccoli P, et al: Video-assisted parathyroidectomy: a series of 85 cases. Chirurgie 1999;124:511-515.

12 Garas G, et al: Radiological considerations and surgical planning in the treatment of giant parathyroid adenomas. Ann R Coll Surg Engl 2015;97:e64-e66.

$\$ 13$ Arora A, et al: The perception of scar cosmesis following thyroid and parathyroid surgery: a prospective cohort study. Int J Surg 2016;25:38-43.

14 Lawrence JW, et al: Visible vs hidden scars and their relation to body esteem. J Burn Care Rehabil 2004;25: 25-32.

15 Ikeda Y, Takami H: Endoscopic parathyroidectomy. Biomed Pharmacother 2000;54:52s-56s.

16 Chung YS, et al: Endoscopic thyroidectomy for thyroid malignancies: comparison with conventional open thyroidectomy. World J Surg 2007;31:2302-2306; discussion 2307-2308.

17 Moorthy K, et al: Dexterity enhancement with robotic surgery. Surg Endosc 2004;18:790-795.

18 Garas G, et al: Evidence-based surgery: barriers, solutions, and the role of evidence synthesis. World J Surg 2012;36:1723-1731.

$>19$ Tolley $\mathrm{N}$, et al: Long-term prospective evaluation comparing robotic parathyroidectomy with minimally invasive open parathyroidectomy for primary hyperparathyroidism. Head Neck 2016;38:E300-E306.

20 Arora A, et al: Comparing transaxillary robotic thyroidectomy with conventional surgery in a UK population: a case control study. Int J Surg 2016;27:110-117.

21 Garas G, Arora A, Tolley N: Robotic surgery of the parathyroid glands; in Grillone G, Jalisi S (eds): Robotic Surgery of the Head and Neck. New York, Springer, 2015, pp 133-146. 
Arora et al.: Robotic Parathyroid Surgery

22 Van Dessel E, et al: Mediastinal parathyroidectomy with the da Vinci robot. Innovations (Phila) 2011;6:262264.

23 Yadav R, et al: Case of the season: ectopic parathyroid adenoma in the pericardium: a report of robotically assisted minimally invasive parathyroidectomy. Semin Roentgenol 2010;45:53-56.

24 Bearelly S, et al: Transoral robotic-assisted surgical excision of a retropharyngeal parathyroid adenoma: a case report. Head Neck 2015;37:E150-E152.

25 Smith MM, et al: Trans-oral robotic surgical excision of an ectopic parathyroid adenoma. J Robot Surg 2016; 10:73-75.

26 Karagkounis G, et al: Robotic surgery for primary hyperparathyroidism. Surg Endosc 2014;28:2702-2707.

27 Noureldine SI, et al: The role of the robotic-assisted transaxillary gasless approach for the removal of parathyroid adenomas. ORL J Otorhinolaryngol Relat Spec 2014;76:19-24.

$28 \mathrm{He}$ Q, et al: Robotic total parathyroidectomy by the axillo-bilateral-breast approach for secondary hyperparathyroidism: a feasibility study. J Laparoendosc Adv Surg Tech 2015;25:311-313.

29 Lee JM, et al: Robotic excision of a huge parathyroid adenoma via a retroauricular approach. J Craniofac Surg 2015;26:e55-e58.

30 Mohamed HE, et al: Robotic transaxillary and retroauricular parathyroid surgery. Gland Surg 2015;4:420428.

-31 Foley CS, et al: Robotic transaxillary endocrine surgery: a comparison with conventional open technique. Surg Endosc 2012;26:2259-2266.

-32 Garas G, et al: Which hemostatic device in thyroid surgery? A network meta-analysis of surgical technologies. Thyroid 2013;23:1138-1150.

-33 Garas G, et al: Is robotic parathyroidectomy a feasible and safe alternative to targeted open parathyroidectomy for the treatment of primary hyperparathyroidism? Int J Surg 2015;15:55-60.

34 Chung WY: Pros of robotic transaxillary thyroid surgery: its impact on cancer control and surgical quality. Thyroid 2012;22:986-987.

-35 Davis SF, et al: Detection and prevention of impending brachial plexus injury secondary to arm positioning using ulnar nerve somatosensory evoked potentials during transaxillary approach for thyroid lobectomy. Am J Electroneurodiagnostic Technol 2011;51:274-279.

-36 Luginbuhl A, et al: Detection of evolving injury to the brachial plexus during transaxillary robotic thyroidectomy. Laryngoscope 2012;122:110-115.

37 Liu SY, Ng EK: Robotic versus open thyroidectomy for differentiated thyroid cancer: an evidence-based review. Int J Endocrinol 2016;2016:4309087.

38 Drummond MF, et al: Methods for the Economic Evaluation of Health Care Programmes, ed 3. New York, Oxford University Press, 2005.

-39 Garas G, et al: Network analysis of surgical innovation: measuring value and the virality of diffusion in robotic surgery. PLoS One 2017;12:e0183332.

-40 Wong KA, et al: How can cardiothoracic and vascular medical devices stay in the market? Interact Cardiovasc Thorac Surg 2016;23:940-948.

41 Trehan A, Dunn TJ: The robotic surgery monopoly is a poor deal. BMJ 2013;347:f7470.

42 Rao C, Athanasiou T: Cost-effectiveness analysis; in Athanasiou T, et al (eds): Key Topics in Surgical Research and Methodology. London, Springer, 2010, pp 411-420.

43 Garas G, et al: Is there survival benefit from life-long follow-up after treatment for differentiated thyroid cancer? Int J Surg 2013;11:116-121.

44 Meltzer C, et al: Surgeon volume in thyroid surgery: surgical efficiency, outcomes, and utilization. Laryngoscope 2016;126:2630-2639. 